\title{
A CCD Optical Search for Visual Very Low-Mass Companions of Halo Stars
}

\author{
EDUARDO L. MARTIN \& RAFAEL REBOLO \\ Instituto de Astrofísica de Canarias, E-38200, La Laguna, Spain
}

\begin{abstract}
We have begun a search for faint companions to cataloged $G / K$ metal-deficient dwarfs. The main goals of this investigation are:

i) to collect a database of halo M-type stars

ii) to study the frecuency of binary separations in the range $1^{\prime \prime}-50^{\prime \prime}$ for Population II stars

iii) to find some metal-poor very-cool dwarfs that may serve as references to investigate the low-mass end of the halo main sequence.
\end{abstract}

In this paper we present the first results from VRI CCD photometry of a subset (51 stars) of our sample. We have identified 7 possible very low mass companions (angular separations $1^{\prime \prime} .5-30^{\prime \prime}$ ) of halo subdwarfs with $[\mathrm{Fe} / \mathrm{H}]$ in the range from -0.8 to -2.6 .

\section{INTRODUCTION}

The scarcity of visual binaries among high-velocity stars has been known for a long time (Oort 1926). Such a difference between the halo and disk galactic populations may provide valuable information on the mechanisms of binary formation and evolution, e.g., the influence of galactic dynamics and metallicity. A photometric search for halo binaries was made by Carney (1983) with the result of a fraction of binarity $20-25 \%$, which is a factor of $\sim 2.5$ lower than in disk stars. Recently, Latham et al. (1992) have reported an extensive survey for spectroscopic binaries in the Carney-Latham proper-motion sample. They find a frequency of short-period spectroscopic binaries similar to that of the field stars. It remains to be determined whether the frequency of long period binaries among halo stars is the same or not from that of disk stars. Our search is a first attempt to resolve this issue. The main aim of the present work is to identify very low mass companions of halo stars with known metallicity. Previous surveys employed methods (radial velocities, abnormal colors) of limited sensitivity to very low mass companions that are intrisically much fainter than their pimaries. The identification of visual very low mass companions is of great interest because of many reasons. We will enumerate some of them:

1. We can more readily detect faint companions when they can be spatially resolved from the primary. Hence, a survey of wide binaries can be complete down to very low masses.

2. A majority of Population I solar type stars ( 53\%, Abt 1979) have distant stellar companions. It is important to determine the visual binary frequency among their Population II counterparts.

3. Very low mass $\left(\mathcal{M} \sim 0.3-0.1 \mathcal{M}_{\odot}\right)$ stars are the largest known contributors to the disk mass density in the solar neighborhood. The mass distribution of distant companions to disk stars raises towards lower masses (down to 
$0.3 \mathcal{M}_{\odot}$, Abt 1979) reflecting the slope of the initial mass function. Thus, we may obtain valuable information on the initial mass function in the halo from the mass distribution of visual metal-poor binaries.

4. Visual very low mass companions stars can be studied individually in more detail than if they are too close to their primaries. Thus, we may obtain from future observations a sample of VLM metal-deficient stars with well known properties that will serve as reference dataset for studying isolated faint proper-motion stars (Hartwick et al. 1984).

\section{METHOD, OBSERVATIONS AND FIRST RESULTS}

Our sample comes from the halo stars $([\mathrm{Fe} / \mathrm{H}] \leq-1.0)$ with metallicity given by Laird et al. (1988) and by Schuster \& Nissen (1989), plus some additional proper motion stars from Hartwick et al. (1984) and Luyten (1979). The selected stars had to meet three criteria. First, they had to be fainter than $V=10$, to avoid CCD saturation effects. Second, they had to be subdwarfs (main-sequence) stars because giants would completely blur out any lower-mass companion. Third, they had to be observable from La Palma $\left(\delta \geq-40^{\circ}\right)$. We observed about 100 stars in 8 nights from 1991 January to March. The observational strategy for each program star was the following:

i) a number of $2^{\prime} \times 2 \cdot 9$ (spatial resolution $0^{\prime \prime} .3$ per pixel) direct CCD images in the $\mathrm{I}_{C}$ band were taken, adjusting the exposure time to have the primary star unsaturated and saturated,

ii) the unsaturated images were visually inspected for faint companions with angular separations $1^{\prime \prime}-10^{\prime \prime}$, and the saturated images were inspected for fainter companions with separations $10^{\prime \prime}-60^{\prime \prime}$,

iii) if any companion was seen in the I-filter frame at less than $\sim 60^{\prime \prime}$ the star was reobserved through the $\mathrm{R}_{C}$ and $\mathrm{V}$ filters. We used the GEC CCD camera at the Cassegrain focus of the $1 \mathrm{~m}$ Jacobus Kapteyn telescope at La Palma observatory. Instrumental magnitudes were derived via aperture photometry for wide pairs, and via point spread function fitting for close pairs. The conversion to the standard Cousins system was performed through a calibration based on equatorial standards observed each night.

In Table 1 below we present results for the first three nights of observations. We found 10 double systems and 1 triple system out of the 51 stars observed. If all the faint companions listed in the table were physically associated to their primaries, the binary frequency would be $22 \%$, which is very similar to that found by Carney (1983). However, one of the secondaries have inconsistent colors as noted in the table, and four others have companions much fainter (2-5 magnitudes) than expected from their colors and the distance to the primary. Hence, we reject 5 companions as probably optical, and we are left with 7 secondaries out of 51 stars, giving a fraction of only $14 \%$. The secondaries with consistent colors and magnitudes have estimated spectral types of M1 to M5 
(from the color versus spectral type calibration of Bessell 1991). Further observational work on these stars is currently in progress in order to estimate their basic parameters.

TABLE 1. Possible Halo Binaries

\begin{tabular}{|c|c|c|c|c|c|c|c|c|c|}
\hline Name & \multicolumn{2}{|c|}{$\begin{array}{l}V \\
\text { (primary) }\end{array}$} & {$[\mathrm{Fe} / \mathrm{H}]$} & Ref. & Sep. & P.A. & $V-I$ & $\begin{array}{c}R-I \\
\text { condery) }\end{array}$ & $I$ \\
\hline $\begin{array}{l}\text { G090-36 } \\
\text { G113-40 } \\
\text { G009-36 }\end{array}$ & $\begin{array}{l}12.9 \\
11.9 \\
11.9\end{array}$ & $\begin{array}{l}0.7 \\
0.9\end{array}$ & $\begin{array}{l}-1.5 \\
-1.1 \\
-1.1\end{array}$ & $\begin{array}{l}\text { SN } \\
\text { SN } \\
\text { SN }\end{array}$ & $\begin{array}{l}1 " .5 \\
30.0 \\
18.0\end{array}$ & $\begin{array}{l}230^{\circ} \\
108 \\
348\end{array}$ & $1.9 \pm 0.3$ & $0.9 \pm 0.2$ & $\begin{array}{l}14.6: \\
18.2\end{array}$ \\
\hline G115-49 & $\begin{array}{l}11.6 \\
11.6\end{array}$ & $\begin{array}{l}0.6 \\
0.6\end{array}$ & $\begin{array}{l}-1.6 \\
-1.6\end{array}$ & $\begin{array}{l}\text { SN } \\
\text { SN }\end{array}$ & $\begin{array}{l}23.9 \\
32.7\end{array}$ & $\begin{array}{r}83 \\
259\end{array}$ & $\begin{array}{l}2.0 \pm 0.1 \\
2.5 \pm 0.8\end{array}$ & $\begin{array}{l}0.9 \pm 0.1 \\
1.2 \pm 0.3\end{array}$ & $\begin{array}{l}14.2 \\
18.1:\end{array}$ \\
\hline $\begin{array}{l}\text { G116-09 } \\
\text { G117-63 }\end{array}$ & $\begin{array}{l}14.3 \\
13.5\end{array}$ & $\begin{array}{l}0.9 \\
0.7\end{array}$ & $\begin{array}{l}-1.6 \\
-0.8\end{array}$ & $\begin{array}{l}\text { LCL } \\
\text { LCL }\end{array}$ & $\begin{array}{r}10.2 \\
3.6\end{array}$ & $\begin{array}{r}92 \\
156\end{array}$ & & $\begin{array}{l}0.9 \pm 0.1 \\
0.2 \pm 0.1\end{array}$ & $\begin{array}{l}16.4 \\
15.6\end{array}$ \\
\hline G119-32 & 10.3 & 0.7 & -1.5 & SN & 29.9 & 210 & & $1.4 \pm 0.2$ & 16.2 \\
\hline $\begin{array}{l}\text { LHS 2500 } \\
\text { G176-46 }\end{array}$ & $\begin{array}{l}18.9 \\
13.0\end{array}$ & $\begin{array}{l}2.3 \\
1.0\end{array}$ & $\begin{array}{l}-2.0: \\
-1.7\end{array}$ & $\begin{array}{l}\text { HCM } \\
\text { LCL }\end{array}$ & $\begin{array}{l}6.3 \\
4.7\end{array}$ & $\begin{array}{r}90 \\
153\end{array}$ & $2.2 \pm 0.1$ & & $\begin{array}{l}19.8: \\
15.8\end{array}$ \\
\hline G059-24 & 12.0 & 0.6 & -2.6 & LCL & 15.2 & 58 & $2.5 \pm 0.2$ & $1.2 \pm 0.1$ & 17.2 \\
\hline G066-22 & 10.7 & 0.8 & -1.0 & SN & 3.7 & 250 & $1.8 \pm 0.1$ & & 12.3: \\
\hline
\end{tabular}

Notes: The photometry is referred to the standard Cousins system. The uncertainty in the quoted I magnitudes is $\mathbf{s} 0.1$, except for those marked with ":" for which it is $\sim 0.2$. The $I$ band frame of G009-36 could not be used. The $(V-R)$ color of the primary is 0.5 and that of the secondary is $(V-R)_{8}=2.1 \pm 0.4$. The $V$ magnitude of the secondary is $20.1 \pm 0.2$.

Sources of $[\mathrm{Fe} / \mathrm{H}]: \mathrm{SN}=$ Schuster \& Nissen (1989), LCL = Laird, Carney \& Latham (1988), HCM = Hartwick, Cowley \& Mould (1984).

\section{ACKNOWLEDGMENTS}

This work is based on observations made with the Jacobus Kapteyn Telescope, operated on the island of La Palma by the Royal Greenwich Observatory in the Spanish Observatorio del Roque de los Muchachos of the Instituto de Astrofísica de Canarias.

\section{REFERENCES}

Abt, H.A. $1979, A J, 84,1591$

Bessell, M.S. 1991, $A J, 101,662$

Carney, B.W. 1983, AJ, 88, 623

Hartwick, F.D., Cowley, A.P., \& Mould, J.R. 1984, ApJ, 286, 269

Laird, J.B., Carney, B.W., \& Latham, D.W. 1988, $A J, 95,1843$

Latham, D.W., Mazeh, T., Stefanik, R.P., Carney, B.W., \& Torres, G. 1992, these proceedings

Luyten, W.J. 1979, LHS Catalog, ed. University of Minnesota

Oort, J.H. 1926, Groningen Publ., 40

Schuster, W.J. \& Nissen, P.E. 1989, à, 222, 69 